\title{
Focusing of Tandem Bistatic SAR Data Using Range Doppler Algorithm
}

\author{
Qisong $\mathrm{Wu}^{\dagger}$, Yimin D. Zhang ${ }^{\dagger}$, Moeness G. Amin ${ }^{\dagger}$, and Braham Himed ${ }^{\ddagger}$ \\ $\dagger$ Center for Advanced Communications, Villanova University, Villanova, PA 19085, USA \\ $\ddagger$ RF Technology Branch, Air Force Research Lab (AFRL/RYMD), WPAFB, OH 45433, USA
}

\begin{abstract}
In this paper, we develop a range Doppler algorithm (RDA) for the tandem bistatic synthetic aperture radar (BiSAR) configuration. The key step is to use an analytical tandem bistatic spectrum. The proposed algorithm is able to handle reasonably high squint by compensating secondary range compression in the two-dimensional wavenumber domain, and is more robust to focus bistatic data compared with Neo's RDA based on the spectrum of method of series reversion (MSR) in the tandem configuration particularly when the baseline-range ratio is large. The effectiveness of the proposed algorithm is verified by simulations.
\end{abstract}

\section{INTRODUCTION}

Bistatic synthetic aperture radar (BiSAR) is characterized by separate locations of the transmitter and the receiver. Because of its many advantages over monostatic SAR such as bistatic imaging, cross-track and along-track interferometry, and reduced vulnerability [1], BiSAR has gained much attention in recent years. As one of the translational invariant (TI) configurations, the tandem case, in which the transmitter and the receiver move along an identical trajectory, has a simple formation structure and can be easily accomplished in practice. The practical bistatic system (TanDEM-X) of such configuration has been shown to acquire well-focused images and interferograms [2].

BiSAR imaging processing is more involved than its monostatic counterpart due to the considerable separation between the transmitter and the receiver. Although time-domain imaging algorithms, such as back projection [3] and its extended fast back projection algorithm [4], can be used to focus bistatic data very well, they are computationally intensive. Alternatively, fast imaging algorithms in the frequency domain such as bistatic range migration algorithm (RMA) [5], bistatic range Doppler algorithm (RDA) [6] are developed for bistatic data processing which uses an approximate point target (PT) spectrum. Unlike monostatic SAR, the BiSAR range history is the sum of two square roots, referred to as double-squareroot (DSR) term, which makes it difficult to use the points of stationary phase (PSP) to acquire a close-form PT spectrum. Three typical bistatic spectrum estimation techniques including dim move out (DMO) [7], Loffeld' bistatic formula (LBF) [8], and method of series reversion (MSR) [9] were

\footnotetext{
The work of Q. Wu, Y. D. Zhang, and M. G. Amin was supported in part by a subcontract with Defense Engineering Corporation for research sponsored by the Air Force Research Laboratory under Contract FA8650-12-D-1376 and by a subcontract with Dynetics, Inc. for research sponsored by the Air Force Research Laboratory under Contract FA8650-08-D-1303.
}

proposed to solve this problem. Several frequency-domain imaging algorithms such as bistatic RDA, Omega-K algorithm [5], and non-linear chirp scaling (NCS) algorithm [10] have been developed over the years. All these algorithms can handle bistatic data very well when the ratio between the baseline and bistatic range history is small. As the ratio increases, the bistatic characteristic in the range history will become prominent. In this case, the above algorithms do not focus the bistatic data well. The main reason is that the accuracy of these spectra is sensitive to the baseline-range ratio, especially the DMO spectrum and the LBF spectrum. As the ratio increases, the accuracy of these two spectra degrades drastically [11]. As a result, it is expected that algorithms based on these spectra will not perform well in the bistatic case with a large ratio.

In contrast to those approximate spectra whose accuracy is affected by the baseline-range ratio, we derived an accurate and analytical bistatic PT spectrum, analyze the relationship between this accurate spectrum and other three spectra including DMO, LBF and MSR spectrum, and verify that these three spectra are approximations of the analytical spectrum in the bistatic tandem configuration [11], [12]. In this paper, and extending the previous works, we develop a range Doppler algorithm based on the analytical bistatic PT spectrum to focus bistatic data. The proposed algorithm is able to obtain wellfocused images in the reasonably high squint bistatic case by compensating the second range compression (SRC) term in the two-dimension (2-D) wavenumber domain and is more robust to the baseline-range ratio.

This paper is organized as follows. In Section II, an exact analytical bistatic PT spectrum is introduced. In Section III, a bistatic RDA is proposed. Simulation results are provided to demonstrate the effectiveness of the proposed algorithm in Section V. Section VI serves as a conclusion of the paper.

\section{Analytical Tandem Bistatic PT Spectrum}

In SAR systems, a linear chirp signal with a wide bandwidth $B$ is transmitted to acquire high resolution images. After demodulation to the baseband, the received echo signal can be expressed in terms of the range time and azimuth range $X=v t_{m}$ domain with platform velocity $v$ and slow time $t_{m}$,

$$
\begin{aligned}
s(\tau, X)= & w_{r}\left[\tau-\frac{R(X)}{c}\right] w_{a z}(X) \exp \left[-j 2 \pi \frac{R(X)}{\lambda}\right] \\
& \times \exp \left[j \pi \gamma\left(\tau-\frac{R(X)}{c}\right)^{2}\right],
\end{aligned}
$$


where $\tau$ denotes the fast time, $w_{r}$ is the range envelope, $w_{a z}$ is the azimuth envelope, $R(X)=R_{T}(X)+R_{R}(X)$ is the range history from the transmitter to target and then back to the receiver, $\lambda=c / f_{c}$ is the wavelength, $f_{c}$ is the carrier frequency, $c$ is the velocity of electromagnetic wave, and $\gamma$ is the chirp rate.

Performing a range Fourier Transform (FT), we obtain the signal in the range wavenumber and azimuth range domain,

$s\left(\Delta K_{R}, X\right)=W_{r}\left(\Delta K_{R}\right) w_{a z}(X) \exp \left[j \frac{\Delta K_{R}^{2}}{2 b}-j K_{R} R(X)\right]$,

where $W_{r}(\cdot)$ represents the spectral shape of the transmitted pulse in the range wavenumber domain, $K_{R}=4 \pi\left(f_{r}+\right.$ $\left.f_{c}\right) / c=\Delta K_{R}+K_{R c}$ is the range wavenumber, $\Delta K_{R}=$ $4 \pi f_{r} / c, f_{r} \in(-B / 2, B / 2)$ is the range frequency, $K_{R c}$ is the range wavenumber center, and $1 / b=2 \pi \gamma / c^{2}$.

Using the concept of instantaneous Doppler wavenumber [11], [13], we obtain

$$
K_{X}=-K_{R}\left[\frac{\partial R_{T}(X)}{\partial X}+\frac{\partial R_{R}(X)}{\partial X}\right]=K_{R} \sin \theta \cos \beta,
$$

where $\beta=\left(\theta_{T}-\theta_{R}\right) / 2$ is referred to as the half quasi bistatic angle, $\theta=\left(\theta_{T}-\theta_{R}\right) / 2$ is the bistatic squint angle, $\theta_{T}$ and $\theta_{R}$ represent the instantaneous squint angles of the transmitter (Tx) and the receiver (Rx), respectively, as depicted in Fig. 1.

We acquire the bistatic PT spectrum based on (2) and (3) according to the geometry-based bistatic formula (GBF) technique [13],

$$
\begin{aligned}
& S\left(\Delta K_{R}, K_{X}\right)=W_{r}\left(\Delta K_{R}\right) W_{a z}\left(K_{X}\right) \\
& \cdot \exp \left(j \frac{\Delta K_{R}^{2}}{2 b}\right) \exp \left(-j K_{X} X_{n}\right) \\
& \cdot \exp \left[-j\left(R_{B}+h_{x}\right) \sqrt{4 K_{R}^{2} \cos ^{2} \beta\left(\Delta K_{R}, K_{X}\right)-K_{X}^{2}}\right],
\end{aligned}
$$

where $W_{a z}(\cdot)$ is the shape of the azimuth wavenumber spectrum, $K_{X}$ is the azimuth wavenumber, $X_{n}$ is the azimuth position of the point target, $R_{B}$ denotes the closest distance from the platform trajectory to target $P_{n}$, and $h_{x}$ is half the baseline.

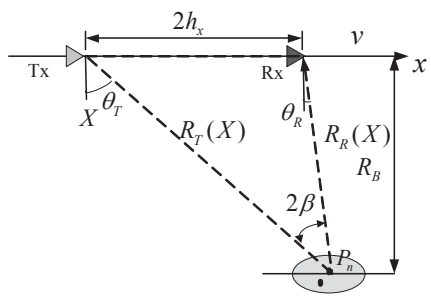

Fig. 1. Bistatic tandem SAR geometry

In Eq. (4), the first exponential phase term represents the range frequency modulation, whereas the second phase term reflects the target azimuth position and the third one represents the bistatic deformation that describes the effect of bistatic operation. It is clear that this spectrum reduces to the monostatic case when the baseline $h_{x}=0$.
It should be pointed out that $\beta\left(\Delta K_{R}, K_{X}\right)$ in the 2-D wavenumber domain is unknown in Eq. (4). Therefore, the spectrum in Eq. (4) is not a bistatic PT spectrum with a closed form unless we acquire the analytical expression of $\beta\left(\Delta K_{R}, K_{X}\right)$, e.g. using numerical computation techniques such as the GBF algorithm [13]. In [11], we derived a close-form expression of $\beta\left(\Delta K_{R}, K_{X}\right)$ in the bistatic tandem configuration, given by

$$
\beta=\arctan \left(-\frac{\sqrt{2 y-2}}{2}+\frac{P}{2}\right),
$$

where

$$
\begin{aligned}
y= & \left(\frac{q}{2}+\sqrt{\left(\frac{q}{2}\right)^{2}+\left(\frac{p}{3}\right)^{3}}\right)^{\frac{1}{3}} \\
& +\left(-\frac{q}{2}-\sqrt{\left(\frac{q}{2}\right)^{2}+\left(\frac{p}{3}\right)^{3}}\right)^{\frac{1}{3}}+\frac{1}{3}, \\
P= & \sqrt{-2-2 y+\frac{8 K_{R}^{2} R_{B}}{h_{x} K_{X}^{2} \sqrt{2 y-2}}}, \\
p= & \frac{4 K_{R}^{2}}{K_{X}^{2}-\frac{4}{3},} \\
q= & \frac{2}{3}\left(1-\frac{4 K_{R}^{2}}{K_{X}^{2}}\right)-\frac{2}{27}-\frac{2 K_{R}^{4} R_{B}^{2}}{h_{x}^{2} K_{X}^{4}} .
\end{aligned}
$$

Substituting Eq. (5) into Eq. (4), a closed-form bistatic PT spectrum can be derived. In [11], we have analyzed the spectrum accuracy and concluded that the DMO, LBF and MSR spectra are merely approximate formulations of the closed-form spectrum.

\section{Bistatic RAnge Doppler Algorithm}

In this section, a bistatic RDA is developed based on the above closed-form spectrum. The processing steps of the bistatic RDA are similar to Neo's method [6], where the secondary range compression (SRC) is compensated for in the 2-D wavenumber domain, whereas the range cell migration correction (RCMC) is done by an interpolator in the range time and azimuth wavenumber domain, and then the azimuth compression operation is performed with a range-dependent phase multiplication. Compared with Neo's RDA method, the proposed algorithm is able to better focus the bistatic data no matter how long the bistatic baseline is. The baseline-range ratio has nearly no effect on the imaging performance.

The development of bistatic RDA starts with the 2-D spectrum (4) of the point target. The phase of spectrum (4) can be decomposed into the following components based on Taylor's expansion with respect to the range wavenumber center $K_{R c}$ up to the quadratic term,

$$
\begin{aligned}
\phi\left(\Delta K_{R}, K_{X}\right) \approx & \phi_{\mathrm{rm}}\left(\Delta K_{R}\right)+\phi_{\mathrm{az}}\left(K_{X}\right)+\phi_{\mathrm{rcm}}\left(K_{R c}, K_{X}\right) \\
& \cdot \Delta K_{R}+\phi_{\mathrm{src}}\left(K_{R c}, K_{X}\right) \Delta K_{R}^{2}+\phi_{\mathrm{res}},
\end{aligned}
$$


where

$$
\begin{aligned}
\phi_{\mathrm{rm}}\left(\Delta K_{R}\right) & =-\frac{\Delta K_{R}^{2}}{2 b}, \\
\phi_{\mathrm{az}}\left(K_{X}\right) & =-\left(R_{B}+h_{x} \tan \beta_{0}\right) \cdot M_{1}, \\
\phi_{\mathrm{rcm}}\left(K_{R c}, K_{X}\right) & =-h_{x} \hat{\beta_{0}} \sec ^{2} \beta_{0} \cdot M_{1} \\
& +\left(R_{B}+h_{x} \tan \beta_{0}\right) \cdot \frac{M_{2}}{M_{1}}, \\
\phi_{\mathrm{src}}\left(K_{R c}, K_{X}\right) & =\frac{M_{4} \cdot M_{1}}{2}+\left(h_{x} \hat{\beta_{0}} \sec ^{2} \beta_{0}\right) \frac{M_{2}}{M_{1}} \\
& -\left[R_{B}\left(1+\rho \tan \beta_{0}\right)\left(\frac{M_{2}^{2}}{2 M_{1}^{3}}-\frac{M_{3}}{2 M_{1}}\right)\right],
\end{aligned}
$$

with

$$
\begin{aligned}
\rho & =h_{x} / R_{B}, \\
M_{1} & =\sqrt{4 K_{R c}^{2} \cos ^{2} \beta_{0}-K_{X}^{2}}, \\
M_{2} & =4 K_{R c}^{2} \cos ^{2} \beta_{0}-2 K_{R c}^{2} \hat{\beta_{0}} \sin \left(2 \beta_{0}\right), \\
M_{3} & =4 \cos ^{2} \beta_{0}-8 K_{R c} \hat{\beta_{0}} \sin \left(2 \beta_{0}\right), \\
& -2 K_{R c}^{2} \hat{\hat{\beta}_{0}} \sin \left(2 \beta_{0}\right)-4 K_{R c}^{2} \hat{\beta}_{0}^{2} \cos \left(2 \beta_{0}\right), \\
M_{4} & =h_{x} \hat{\hat{\beta}}_{0} \sec ^{2} \beta_{0}+2 h_{x} \hat{\beta}_{0}^{2} \tan \beta_{0} \sec ^{2} \beta_{0}, \\
\beta_{0} & =\left.\beta\right|_{K_{R}=K_{R c}}, \hat{\beta}_{0}=\left.\frac{\partial \beta}{\partial K_{R}}\right|_{K_{R}=K_{R c}}, \hat{\hat{\beta}}_{0}=\left.\frac{\partial^{2} \beta}{\partial K_{R}^{2}}\right|_{K_{R}=K_{R c}} .
\end{aligned}
$$

The above phase terms can be interpreted as follows:

(a) The first phase term $\phi_{\mathrm{rm}}\left(\Delta K_{R}\right)$ represents the range modulation, which is dependent only on the range wavenumber. This term can be removed through range compression by multiplying a conjugated phase. This operation is the same as the monostatic case.

(b) The second phase term $\phi_{\mathrm{az}}\left(K_{X}\right)$ represents the azimuth modulation. A conjugated phase is used to perform azimuthmatched filtering. This operation is applied in the range time and azimuth wavenumber domain due to significant range dependence.

(c) The third term $\phi_{\mathrm{rcm}}\left(\Delta K_{R}, K_{X}\right)$ is linearly proportional to the range wavenumber $\Delta K_{R}$ and represents the RCM term. A range direction interpolator is used to correct the RCM in the range time and azimuth wavennumber domain in a similar manner with the monostatic RDA.

(d) The fourth phase term $\phi_{\mathrm{src}}\left(\Delta K_{R}, K_{X}\right)$ represents range/azimuth coupling term and becomes significant for a high squint angle, a fine resolution, or large bistatic baseline. If uncompensated, the coupling term would lead to significant degradation in the resolution. The SRC is compensated in the 2-D wavenumber domain, since the strong dependency exists in the 2-D wavenumber domain. However, this SRC term is weakly range dependent and $\phi_{\mathrm{src}}\left(\Delta K_{R}, K_{X}\right)$ is computed at a specific range called reference range which is always at the swath center. For wider range swath, it may be necessary to segment the scene into multiple range invariance regions whose width is determined by the tolerable quadratic phase error.

(e) The last phase term $\phi_{\text {res }}$ represents the residual phase, including phase terms that are independent of range and azimuth wavenumber and higher-order error phase terms. This phase term has no effect on the focusing processing.
In contrast to the finite truncation of $K_{X}$ in the Taylor expansion used in Neo's method, the proposed algorithm keeps the information of $K_{X}$ without any loss in $\phi_{\mathrm{az}}\left(K_{X}\right), \phi_{\mathrm{rcm}}\left(\Delta K_{R}, K_{X}\right)$ and $\phi_{\mathrm{src}}\left(\Delta K_{R}, K_{X}\right)$. While the losses caused by the truncation operation, are insignificant in a small baseline-range ratio case, they become large as the baseline-range ratio increases and lead to degradation of the focusing quality. This will be demonstrated in the simulations. Compared with the complexity of Neo's RDA method that performs the Taylor expansions in terms of $K_{X}$ and $\Delta K_{R}$, the proposed algorithm only performs the Taylor expansion in terms of $\Delta K_{R}$ and the extra computations of $\hat{\beta}_{0}$ and $\hat{\beta}_{0}$. As such, their complexities are comparative.

Removing the range compression term and the SRC term in the 2-D wavenumber domain, the reference function becomes

$$
\begin{aligned}
\varphi_{r}\left(\Delta K_{R}, K_{X}, R_{s}\right)= & \exp \left[-j \phi_{\mathrm{src}}\left(K_{R c}, K_{X}, R_{s}\right) \Delta K_{R}^{2}\right] \\
& \cdot \exp \left(-j \phi_{\mathrm{rm}}\right),
\end{aligned}
$$

where $R_{s}$ is the reference range from the reference target to the trajectory.

Taking the inverse range FT, we obtain the signal in range time and azimuth wavenumber domain as

$$
S\left(\tau, K_{X}\right)=\omega_{r}\left(\tau-\frac{\phi_{\mathrm{rcm}}}{c}\right) W_{\mathrm{az}}\left(K_{X}\right) \exp \left[j \phi_{\mathrm{az}}\left(K_{X}\right)\right],
$$

where $\omega_{r}$ is the sinc-like compressed envelope and RCM term appears in this envelope. RCMC is performed by a range-depedent interpolation in this step. The RCMC operation straighten the trajectories so that they become parallel to the azimuth wavenumber axis.

The last step is the azimuth compression. The reference function becomes

$$
\varphi_{a z}\left(K_{X}\right)=\exp \left[-j \phi_{\mathrm{az}}\left(K_{X}\right)\right] .
$$

After this step, the inverse azimuth FT is then performed to transform the data into the complex image domain.

\section{ALgORITHM ANALYSIS}

In the previous section, a bistatic RDA method based on the analytical PT spectrum is developed. As we discussed above, the coupling phase term (11), if not properly compensated, will lead to significant degradation in the image resolution. In the RDA algorithm, the SRC compensation operation is performed independent of range. As the range region size increases, the focusing quality of points in the margin degrades drastically, especially in the case with a large baseline-range ratio. It is thus necessary to analyze the relationship between the baseline-range ratio and the range region size in this algorithm.

Focusing on the SRC compensation operation in (12), we compensate the range-dependent SRC phase term by a reference phase term with reference range $R_{s}$. The quadratic phase error (QPE) due to range region size is expressed by neglecting the second and higher-order error phases, i.e.,

$$
\epsilon\left(\rho, \Delta R_{B}\right) \approx \frac{\partial \phi_{\mathrm{src}}\left(K_{R c}, K_{X}, \rho\right)}{\partial R_{B}} \Delta R_{B} \Delta K_{R}^{2}
$$


where $\Delta R_{B}=R_{B}-R_{s}$. The maximum value of $\mathrm{QPE}$ is given by

$$
\epsilon_{\max }\left(\rho, \Delta R_{B}\right)=\frac{\partial \phi_{\mathrm{src}}\left(K_{R c}, K_{X}, \rho\right)}{\partial R_{B}} \Delta R_{B}(B / c)^{2} .
$$

Within a range processing block, the QPE should be limited by $\nu \pi$, where $\nu$ is normally set to 0.25 . According to (16), the maximum range offset can be determined via the condition

$$
\Delta R_{B, \max }=\frac{\nu \pi c^{2}}{B^{2}}\left[\frac{\partial \phi_{\mathrm{src}}\left(K_{R c}, K_{X}, \rho\right)}{\partial R_{B}}\right]^{-1} .
$$

It is clear that the tolerable range offset $\Delta R_{B}$ is inversely proportional to the transmitted bandwidth $B$. Fig. 2 shows the relationship between $\Delta R_{B, \max }$ and the ratio $\rho$ when $B=80$ $\mathrm{MHz}$. It is clear that the proposed algorithm can well focus these targets for a range region size of $350 \mathrm{~m}$ even when the baseline-range ratio is up to 1 . However, the tolerable range region size in the Neo's RDA method is only $20 \mathrm{~m}$. The main reason is that the accuracy of MSR spectrum degrades and the losses due to the truncation operation become larger as the baseline-range ratio increases, and thus the tolerable range region size in Neo's RDA based on the MSR spectrum becomes smaller. The LBF and DOM spectra completely fail in such a large ratio. They cannot even focus the reference target [11]. Their region sizes become comparative as the ratio decreases.

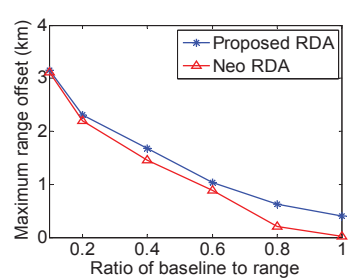

Fig. 2. Maximum range offset versus the ratio of baseline to range.

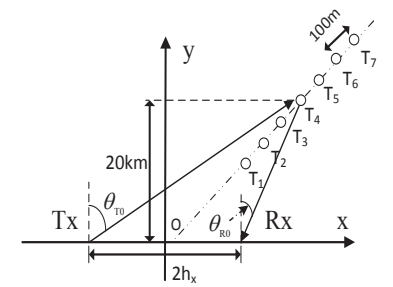

Fig. 3. Geometry of the bistatic simulation and scene arrangement.

\section{SIMULATIONS}

In this section, two simulations are performed to verify the feasibility of the proposed algorithm and effectiveness of the analysis above. Simulation parameters are given in Table I. The oversampling rate is 1.5 in range and 1.8 in azimuth; rectangular weighting is adopted in both range and azimuth processing. Point target arrangement in the scene is shown in Fig. 3. Seven point targets (labeled as T1-T7) are illuminated by a composite bistatic beam. The separation between adjacent point targets is $100 \mathrm{~m}$. The target $\mathrm{T} 4$ is assumed to be the reference target, and the closest range from $\mathrm{T} 4$ to the trajectory is $20 \mathrm{~km}$.

In the first case, the baseline is $8 \mathrm{~km}$ and the ratio $\rho$ is 0.4. An appreciable amount of squint angle with transmitter squint angle $\theta_{T 0}$ of $34.99^{\circ}$ and receiver squint angle $\theta_{R 0}$ of $16.70^{\circ}$ is assumed to introduce a severe SRC term. The RCMs and SRC compensation and range cell correction in the range time and azimuth wavenumber domain are shown in Fig. 4. RCMs caused by the bistatic squint model are very clear in Fig. 4(a) and Fig. 4(b). SRC is compensated to remove the range/azimuth coupling and the range interpolator is used to remove these RCMs and the results after correction are shown in Fig. 4(c). Taking the azimuth compression and inverse FT, we obtain the complex image as shown in Fig. 5(a). The contour plots of reference target $\mathrm{T} 4$ and the farthest target T7 are shown in Fig. 5(b). Range impulse responses, azimuth impulse responses and their quality measurements of two point targets (T4 and T7) are shown in Fig. 6. The impulse response width (IRW) is 1.31 cells in range and 1.63 cells in azimuth, which meets the theoretical limits in range $(1.33 / 1.5=0.88)$ and in azimuth $(1.63 / 1.8=0.90)$ for rectangular weighting. Furthermore, the sidelobe levels agree with the theoretical values of $-13.3 \mathrm{~dB}$ and $-10 \mathrm{~dB}$ for peak sidelobe ratio (PSLR) and integrated sidelobe ratio (ISLR), respectively. As such, we conclude that the proposed RDA algorithm is able to focus data in the tandem bistatic configuration very well.

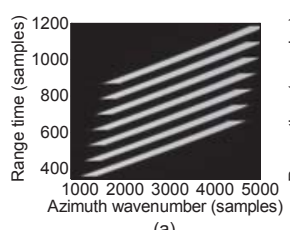

(a)

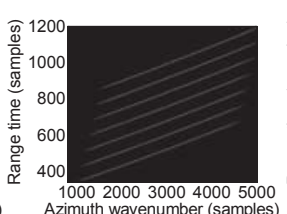

(b)

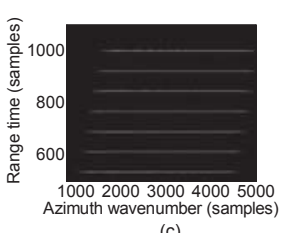

(c)
Fig. 4. Processing of SRC compensation and MCMC operation (a) Data after azimuth FT . (b) Data after SRC compensation (c) Data after MCMC.

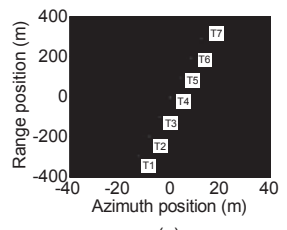

(a)

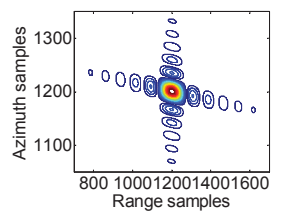

(b)

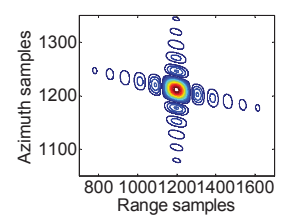

(c)
Fig. 5. Point targets imaging. (a) Point targets focused using Proposed RDA. (b) Contour plot of reference target (T4) based on the proposed RDA. (c) Contour plot of the farthest target (T7) based on the proposed RDA.

In the second simulation, it is verified that the proposed algorithm is more robust compared with Neo's RDA as the ratio of baseline-range increases. To analyze the effect of baseline on the proposed RDA algorithm, the simulation with a longer baseline (20 km and $\rho=1.0)$ is performed. It is evident that the differences in both the range and azimuth impulse responses are negligible for both $\mathrm{T} 4$ and $\mathrm{T} 7$, as shown in Fig. 6. For comparison purposes, the range impulse responses, the azimuth impulse responses, and the focusing quality measurements of two point targets (T4 and T7) are shown in Fig. 7 based on Neo's bistatic RDA method. The reference target $\mathrm{T} 4$ has excellent focused qualities in both range and azimuth impulse responses in both cases, whereas the performance of the range response of the farthest target T7 drastically degrades as the baseline-range ratio increases. Its PLSR and ISLR in range degrade up to $-7.89 \mathrm{~dB}$ and 
TABLE I

SIMULATION PARAMETERS

\begin{tabular}{|c|c|c|c|c|c|c|c|c|}
\hline & Baseline & Squint $\theta_{T 0}$ & Squint $\theta_{R 0}$ & Center frequency & Range bandwidth & Sample rate & Velocity & PRF \\
\cline { 1 - 8 } Case 1 & $8 \mathrm{~km}$ & $34.99^{\circ}$ & $16.70^{\circ}$ & \multirow{2}{*}{$10 \mathrm{GHz}$} & $80 \mathrm{MHz}$ & \multirow{2}{*}{$120 \mathrm{MHz}$} & \multirow{2}{*}{$150 \mathrm{~m} / \mathrm{s}$} & $600 \mathrm{~Hz}$ \\
\hline Case 2 & $20 \mathrm{~km}$ & $45.00^{\circ}$ & $0^{\circ}$ & & & \\
\hline
\end{tabular}

$-6.36 \mathrm{~dB}$, respectively, as are shown in Fig. 7(b). The reason behind this is that Neo's bistatic RDA method was developed based on the MSR's spectrum which is approximate and its accuracy is sensitive to the baseline-range ratio. Therefore, it is expected that Neo's method will not perform well when the baseline-range ratio is large. As a result, the proposed RDA method is more robust to focus the bistatic tandem data and the baseline-range ratio has negligible impact on the imaging results.
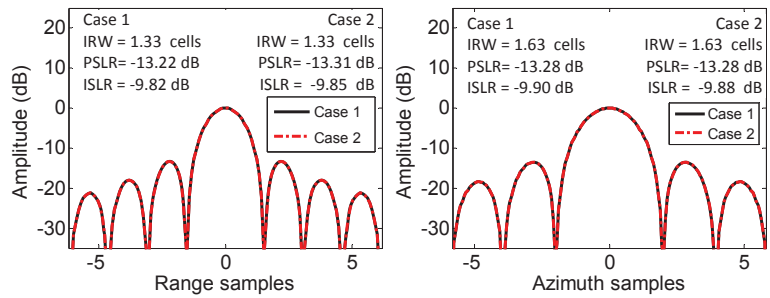

(a)
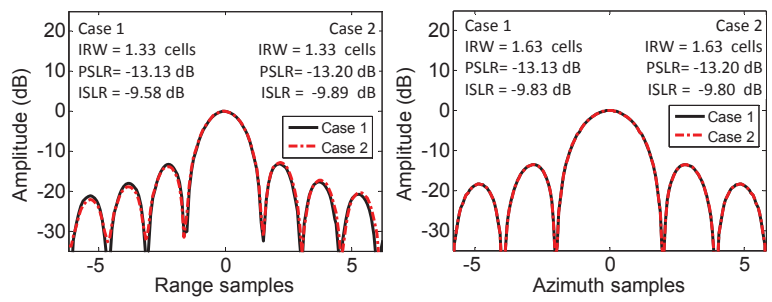

(b)

Fig. 6. Plots of range and azimuth impulse response based on the proposed algorithm. (a) Plots of range and azimuth impulse response for T4. (b) Plots of range and azimuth impulse response for T7.

\section{CONCLUSION}

An analytical spectrum-based bistatic RDA algorithm is developed to focus SAR data in the tandem configuration. Unlike existing algorithms, the proposed RDA algorithm is insensitive to the baseline-range ratio and is thus more robust in the bistatic tandem case. As a result, The proposed technique is effective when the bistatic SAR data is acquired with a large baseline in the tandem configuration.

\section{REFERENCES}

[1] N. J. Willis, Bistatic Radar. Artech House, 1991.

[2] M. Rodriguez-Cassola, P. Prats, D. Schulze, N. Tous-Ramon, U. Steinbrecher, L. Marotti, M. Nannini, M. Younis, P. Lopez-Dekker, M. Zink, A. Reigber, G. Krieger, and A. Moreira, "First bistatic spaceborne SAR experiments with TanDEM-X," IEEE Geosci. Remote Sens. Lett., vol. 9, no. 1, pp. 33-37, 2012.

[3] B. Barber, "Theory of digital imaging from orbit synthetic aperture radar," Int. J. Remote Sens., vol. 6, no. 6, pp. 1009-1057, 1985.

[4] M. R. Cassola, P. Prats, G. Krieger, and A. Moreira, "Efficient timedomain image formation with precise topography accommodation for general bistatic SAR configurations," IEEE Trans. Aerospace Electron. Syst., vol. 47, no. 4, pp. 2949-2966, 2010.
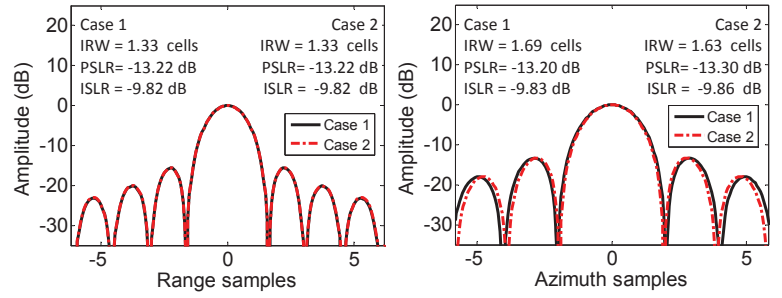

(a)
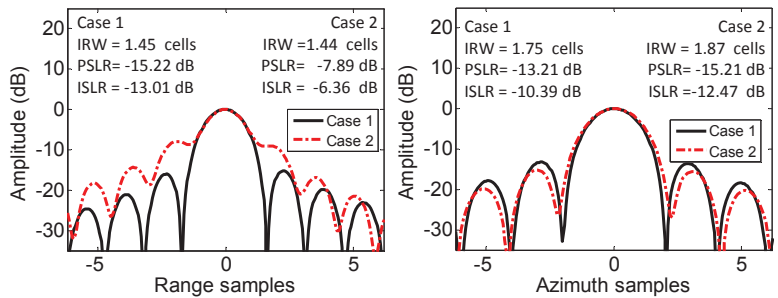

(b)

Fig. 7. Plots of range and azimuth impulse response based on Neo's RDA method. (a) Plots of range and azimuth impulse response for T4. (b) Plots of range and azimuth impulse response for T7.

[5] B. Liu, T. Wang, Q. Wu, and Z. Bao, "Bistatic SAR data focusing using an Omega-K algorithm based on method of series reversion," IEEE Trans. Geosci. Remote Sens., vol. 47, no. 8, pp. 2899-2912, 2009.

[6] Y. L. Neo, F. H. Wong, and I. G. Cumming, "Processing of azimuthinvariant bistatic sr data using the range doppler algorithm," IEEE Trans. Geosci. Remote Sens., vol. 46, no. 1, pp. 14-21, 2008.

[7] D. D. Aria, M. Guarnieri, and F. Rocca, "Focusing bistatic synthetic aperture radar using dip move out," IEEE Trans. Geosci. Remote Sens., vol. 42, no. 7, pp. 1362-1376, 2004.

[8] O. Loffeld, H. Nies, V. Peters, and S. Knedlik, "Models and useful relations for bistatic SAR processing," IEEE Trans. Geosci. Remote Sens., vol. 42, no. 10, pp. 2031-2038, 2004.

[9] Y. L. Neo, F. H. Wong, and I. G. Cumming, "A two-dimensional spectrum for bistatic SAR processing using series reversion," IEEE Geosci. Remote Sens. Lett., vol. 4, no. 1, pp. 93-96, 2007.

[10] F. H. Wong, I. G. Cumming, and Y. L. Neo, "Focusing bistatic SAR data using the nonlinear chirp scaling algorithm," IEEE Trans. Geosci. Remote Sens., vol. 46, no. 9, pp. 2493-2505, 2008.

[11] Q. Wu, M. Xing, H. Shi, X. Hu, and Z. Bao, "Exact analytical twodimensional spectrum for bistatic synthetic aperture radar in tandem configuration," IET Radar Sonar Navig., vol. 5, no. 3, pp. 349-360, 2011.

[12] S. Chen, Q. Wu, P. Zhou, M. Xing, and Z. Bao, "A new look at the Loffeld's bistatic formula in tandem configuration," IEEE Geosci. Remote Sens. Lett., vol. 9, no. 1, pp. 710-714, 2010.

[13] Z. Zhang, M. Xing, J. Ding, and Z. Bao, "Focusing parallel bistatic SAR data using the analytic transfer function in the wavenumber domain," IEEE Trans. Geosci. Remote Sens., vol. 45, no. 11, pp. 3633-3645, 2007. 